\title{
The Value of Calcium Dobesilate in the Treatment of Diabetic Retinopathy
}

\author{
A Controlled Clinical Trial \\ H.-W. Larsen ${ }^{1}$, E. Sander ${ }^{2}$, and R. Hoppe ${ }^{1}$ \\ Department of Ophthalmology ${ }^{1}$, Gentofte Hospital, University of Copenhagen, School of Medicine, and Niels Steensens Hospital ${ }^{2}$, \\ Gentofte, Denmark
}

\begin{abstract}
Summary. The effect of calcium dobesilate on capillary resistance and background retinopathy was compared with placebo in a double-blind cross-over trial on 18 diabetics. Each treatment lasted 8 months. The trial produced no evidence in favour of a significant beneficial effect of calcium dobesilate on the capillary resistance in diabetics or on the course of the diabetic retinopathy.
\end{abstract}

Key words: Calcium dobesilate, Doxium, diabetic retinopathy, fluorescein angiography, capillary resistance, diabetes mellitus.

In 1969 Sévin \& Cuendet [10] reported on the use of a synthetic drug, calcium dobesilate (Doxium ${ }^{\circledR}$, calcium 2.5 dihydroxybenzene sulphonate) in diabetics with retinopathy and reduced capillary resistance. Other papers have since appeared on the same subject. Ca-dobesilate was found to improve the capillary resistance in diabetics as measured on the bulbar conjunctiva $[10,12,9]$ and by measurements on the skin [2]. Rasch [8], however, found no effect of Ca-dobesilate on the skin capillary resistance. Several groups $[10,9,2,3]$ found that Ca-dobesilate reduced the number of retinal haemorrhages in diabetics.

By means of fluorescein angiography it was found that $\mathrm{Ca}$-dobesilate reduced the pathological permeability of the retinal capillaries $[11,12,6,4$, 3], whereas Freyler [5] found that Ca-dobesilate was only of value in the treatment of early stages of simple diabetic retinopathy, with small hard exudates.

No influence of $\mathrm{Ca}$-dobesilate was found on the course of proliferative diabetic retinopathy $[11,3]$.

In order to estimate the value of calcium dobesilate in the treatment of diabetic retinopathy, a double-blind cross-over trial was started in 1972 on diabetics with background retinopathy.

\section{Material}

25 consecutive diabetics with background retinopathy suitable for the investigation entered the trial. They were randomly allocated to treatment with either Ca-dobesilate or placebo. The Cadobesilate group received tablets of $250 \mathrm{mg}$ three times a day for eight months, and continued thereafter with placebo three times a day for another eight months. The placebo group started with identical placebo tablets three times a day for eight months, and continued thereafter with Ca-dobesilate tablets of $250 \mathrm{mg}$ three times a day for another eight months.

None of the investigators $\mathrm{knew}$ if a patient was on Ca-dobesilate or on placebo. The code was not broken until the end of the trial when the results for each patient and each period had been evaluated.

Seven subjects dropped out of the trial. In 4 an initial satisfactory fluorescein angiography could not be obtained for various reasons and the patients therefore never entered the trial. In one case the patient ceased taking the tablets after 4 months. The remaining two patients were taken out of the trial because one developed leukaemia (on placebo) and the other an occlusion of the central retinal artery in one eye (on placebo).

The group was therefore reduced to 18 diabetics who completed the trial.

12 were males and 6 females. The average age was 48 years (range $20-72$ years). The average duration of diabetes was 15.5 years (range 1-33 years). Fourteen were treated with insulin, 3 with oral antidiabetics and one with diet only. The diabetes was satisfactorily regulated in all cases except 
one. Three had hypertension, one nephropathy, one chronic cystitis and 2 neuropathy.

\section{Methods}

A medical evaluation, including measurements of skin capillary resistance, was performed prior to the trial and repeated after 8 and 16 months. The skin capillary resistance was measured with the negative suction cup technique [1].

A suction cup with an inner diameter of $7 \mathrm{~mm}$ and an outer diameter of $13 \mathrm{~mm}$ was used. Initially a negative pressure of $50 \mathrm{~mm} \mathrm{Hg}$ was applied and continued for one minute. If no petechiae appeared the suction pressure was increased in steps of $25 \mathrm{~mm}$ $\mathrm{Hg}$ until one or more petechiae appeared. The pressure at which this occurred was noted as the capillary resistance of that particular spot. The whole procedure was repeated three times on the anteromedial side of the upper arm and the capillary resistance was defined as the mean value of three measurements. All measurements of the skin capillary resistance were performed at a room temperature of $20-23^{\circ} \mathrm{C}$ and the patients were in the room for about half an hour before the measurements were undertaken.

Prior to the trial an eye examination including fundus photography and fluorescein angiography was performed. This was repeated after 8 and 16 months.

Five standard colour fundus photographs (macular area, disc area, areas above, below and nasal to the disc) were taken of both eyes. For fluorescein angiography, $5 \mathrm{ml}$ of a 10 per cent solution of sodium fluorescein was injected into a cubital vein within 3-4 sec. The angiograms were recorded with a Topcon fundus camera provided with standard filters on a Kodak Tri-X film. Photographs were taken at 1-2 second intervals in the early phases and at 10,20 and 30 minutes after injection. The right eye was used whenever possible. In the early phases the macular area was photographed. In the late phases, all areas corresponding to the colour fundus photographs were also recorded.

The assessment of the fundus photographs and the fluorescein angiograms was carried out by comparison and quantitative analysis of the pathological changes (i. e. microaneurysms, retinal haemorrhages and exudates, hyperpermeability and non-perfused areas) in the above mentioned areas at each control examination.

\section{Results}

11 started with placebo and 7 with Ca-dobesilate. The groups were fully comparable with respect to all recorded variables.
2 .

The individual results are shown in Table 1 and

\section{Skin Capillary Resistance}

The initial median value for the 18 diabetics was $171 \mathrm{~mm} \mathrm{Hg}$ (range 108-258). After treatment with Ca-dobesilate for 8 months the median increase was $6 \mathrm{~mm} \mathrm{Hg}$ (95 per cent confidence limits from -25 to +42 ). After placebo treatment for 8 months the median incrase was $0 \mathrm{~mm} \mathrm{Hg}$ (95 per cent confidence limits from -33 to +41 ). Analysis of variance showed no statistically significant difference between the two forms of treatment $(\mathrm{P}>>0.10)$.

\section{Diabetic Retinopathy}

An evaluation based on fluorescein angiography and fundus photography in one eye (usually the right eye) showed the following: In 13 diabetics the retinopathy was unchanged during both $\mathrm{Ca}$-dobesilate and placebo treatment. In 3 diabetics the retinopathy deteriorated during both Ca-dobesilate and placebo treatment, and two progressed into proliferative retinopathy during $\mathrm{Ca}$-dobesilate treatment. In 2 diabetics the retinopathy deteriorated during placebo treatment and one of these showed improvement during Ca-dobesilate treatment. Differences between $\mathrm{Ca}$-dobesilate and placebo were not statistically significant.

An evaluation based on both eyes showed $3 \mathrm{Ca}$ dobesilate preferences, and 2 placebo preferences, while 9 were unchanged and 4 deteriorated during both treatments. These differences are not statistically significant. Ca-dobesilate preference denotes cases with either improvement on Ca-dobesilate and deterioration or constant findings on placebo, or unchanged findings on $\mathrm{Ca}$-dobesilate with deterioration on placebo.

An evaluation based on fluorescein angiography alone showed $3 \mathrm{Ca}$-dobesilate preferences and 4 placebo preferences, while 6 were unchanged, 3 deteriorated and 2 improved during both treatments. These differences are not statistically significant.

An evaluation based on microaneurysms alone showed $3 \mathrm{Ca}$-dobesilate preferences and 2 placebo preferences, while 9 were unchanged, 3 deteriorated and one improved during both treatments. These differences are not statistically significant.

An evaluation based on retinal hemorrhages alone showed Ca-dobesilate better than placebo, but not significantly $(P>0.10)$. An evaluation based on hard exudates alone showed Ca-dobesilate statistically significantly better than placebo $(P<0.05)$.

Too much importance, however, should not be placed on the evaluations based only on retinal hemorrhages and hard exudates, partly for statisti- 
cal, partly for methodological reasons. Thus 2 patients who were placebo preferences by evaluation of retinal haemorrhages became $\mathrm{Ca}$-dobesilate preferences by evaluation of hard exudates, whereas one patient who was a Ca-dobesilate preference by evaluation of retinal haemorrhages became a placebo preference by evaluation of hard exudates.

Generally only minor changes took place in the retinopathy during the trial. Two points worth stressing are that 2 diabetics developed proliferative retinopathy during $\mathrm{Ca}$-dobesilate treatment and that the diabetic retinopathy remained active in all patients during both periods of treatment.

\section{Discussion}

Capillary resistance is usually reduced in long-term diabetics. This may be one of the causal factors in the development of retinal haemorrhage. A drug which can normalize the capillary resistance could therefore be of value in the treatment of diabetic retinopathy.

Uncontrolled and partly controlled studies on the capillary resistance of the bulbar conjunctiva $[10,11,12,9]$ and the skin [2] have shown that $\mathrm{Ca}-$ dobesilate in doses of 500-750 mg daily has a beneficial effect on the capillary resistance in diabetics. The rise in capillary resistance was constant during at least 6 months. In these papers however, there is no information about the temperature at which the measurements took place nor about the validity of the test used on the bulbar conjunctiva.

Rasch [8] employing a double-blind clinical trial and using $500 \mathrm{mg}$ Ca-dobesilate daily, could not find any effect of the drug on skin capillary resistance. The average age of her patients was 48 years. In the present trial Ca-dobesilate in doses of $750 \mathrm{mg}$ daily for 8 months also had no effect on skin capillary resistance. Both in Rasch's trial and in our own the capillary resistance was measured under standardized conditions.

Based upon these two controlled trials, it seems justified to conclude that Ca-dobesilate does not influence the capillary resistance in diabetics.

An improvement in the course of the diabetic retinopathy by $\mathrm{Ca}$-dobesilate was described in uncontrolled $[10,11,12,3,2]$ and in controlled studies [9, $4,6]$. A highly significant effect of Ca-dobesilate on the permeability of the retinal capillaries in diabetics with background retinopathy $[11,4,6]$ and a significant regression and/or stabilisation of hard exudates by Ca-dobesilate [4, 3] was found. Freyler [5], however, in a later paper stated that only small, single, hard exudates were influenced by $\mathrm{Ca}$-dobesi-

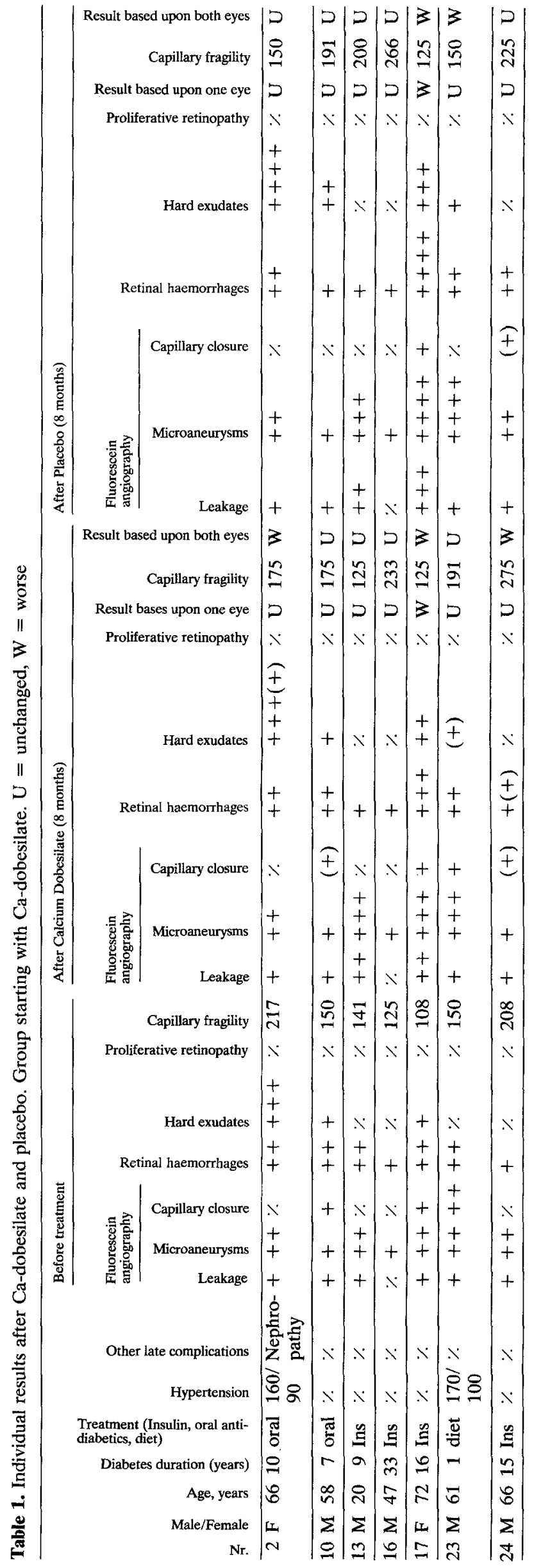




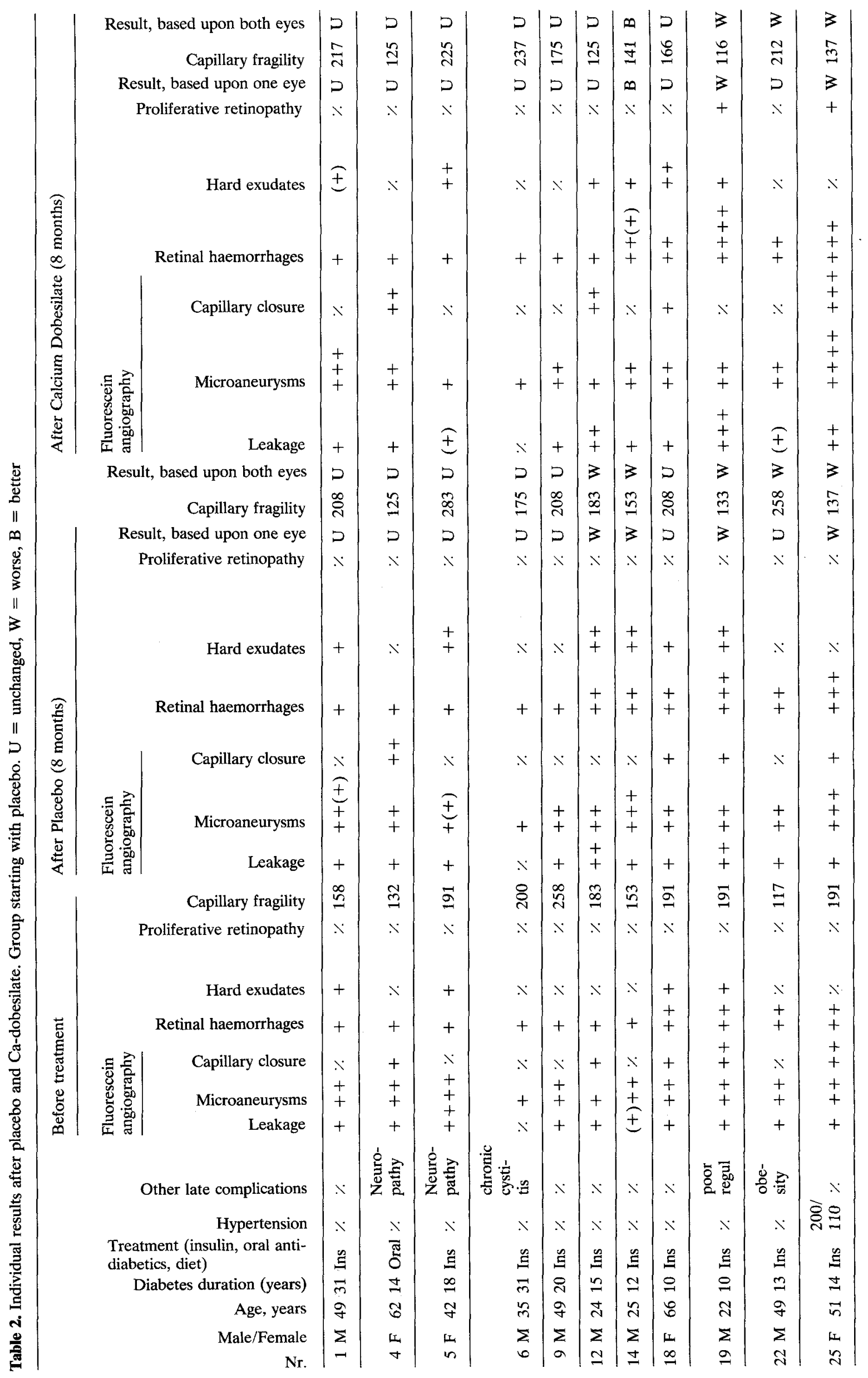


late, while larger plaques of exudates can be destroyed by photocoagulation only. A striking regression of microaneurysms was reported by two groups $[4,6]$ and no effect by 2 others [3, 9]. No influence of $\mathrm{Ca}$-dobesilate on proliferative diabetic retinopathy was observed $[11,12,3]$. In some reports $[10,11,2,4,6,3]$ the average age was $58-61$ years, while ages are not listed in others $[12,9]$. The blood pressure is indicated in one report [4] only.

In the present trial, the main course of the retinopathy was not significantly influenced by $\mathrm{Ca}$ dobesilate and the retinopathy remained active in all cases during both Ca-dobesilate and placebo treatment. Some regression of hard exudates was observed during Ca-dobesilate treatment, but as previously stated too much importance should not be placed on this evaluation partly for statistical, partly for methodological reasons.

Our patients differ in some respects from the patients in the other trials.

In the present study as well as that of Rasch [8] the average age was 48 years, differing significantly from the average age of 58-61 years in the other trials. It is impossible to determine whether such differences in the mean age could influence the results obtained.

Another difference is the frequent occurrence of hypertension in Freyler's [4] subjects. 23 of 36 diabetics had hypertension and received antihypertensive treatment during the investigation. In the present study of 18 diabetics only two had mild hypertension and one a hypertension with a systolic blood pressure of $200 \mathrm{~mm} \mathrm{Hg}$. None received antihypertensive treatment.

Some of the beneficial changes in leakage and the reduction in the number of microaneurysms in Freyler's material may not be due to Ca-dobesilate but to the antihypertensive treatment.

In a study similar to ours, Kolsted [7] obtained exactly the same results with Ca-dobesilate. This supports the view that the drug does not significantly influence the course of diabetic retinopathy.

\section{Conclusion}

This clinical double-blind cross-over trial on 18 diabetics did not produce evidence in favour of a significant beneficial effect of calcium dobesilate on the capillary resistance in diabetics or on the course of diabetic retinopathy.

Acknowledgements. Ca-dobesilate tablets and placebo tablets were provided by Ercopharm, Vedbæk, Denmark, and the statistical analyses were made by $\mathrm{Bj}$. Andersen.

\section{References}

1. Christensen, J. J., Terkildsen, A. B.: Quantitative measurements of skin capillary resistance in hypophysectomized longterm diabetics. Diabetes 20, 297-301 (1971)

2. Favre, M.: Le traitement de la rétinopathie diabétique et de l'hémorragie récidivante du vitré par le dobésilate de calcium. Ophthalmologica (Basel) 161, 389-393 (1970)

3. Francois, J., Verbraeken, H.: Le dobésilate de calcium dans le traitement de la rétinopathie diabétique. Ann. Oculist. (Paris) 208, 157-160 (1975)

4. Freyler, H.: Microvascular protection with calcium dobesilate (Doxium) in diabetic retinopathy. Ophthalmologica (Basel) 168, 400-416 (1974)

5. Freyler, H.: Die exsudative diabetische Retinopathie. Ophthalmologica (Basel) 170, 518-533 (1975)

6. Freyler, H., Sehorst, W.: Gefäßabdichtung bei diabetischer Retinopathie durch Kalziumdobesilat. Klin. Mbl. Augenheilk. 164, 246-251 (1974)

7. Kolsted, A.: Personal communication (1975)

8. Rasch, R.: Capillary fragility and doxium. Diabetologia 9 , 483-485 (1973)

9. Regnault, F.: Étude de l'action du dobésilate de calcium sur les rétinopathies vasculaires de l'homme. Ann. Oculist. (Paris) 207, 369-374 (1974)

10. Sévin, R., Cuendet, J.F.: Calcium dobesilate in diabetic retinopathy. Ophthalmologica (Basel) 159, 126-135 (1969)

11. Sévin, R., Cuendet, J.F.: The action of calcium dobesilate on capillary permeability in diabetics. Ophthalmologica (Basel) 162, 33-40 (1971)

12. Temprano, J.: Clinical trial of calcium dobesilate, "Doxium", in diabetic retinopathy. Anal. Inst. Barraquer 11, 9-16 (1973)

Received: August 30, 1976, and in revised form: December 3, 1976

H.-W. Larsen, M. D.

University of Copenhagen

School of Medicine

Department of Ophthalmology

Gentofte Hospital

Niels Andersensvej

DK-2900 Hellerup

Denmark 